\title{
Regiocontrolled Oxidative C-C Coupling of Dienol Ethers and 1,3-Dicarbonyl Compounds
}

\author{
Xiaoguang Liu, Xiaohong Chen, and Justin T. Mohr*
}

Department of Chemistry, University of Illinois at Chicago, 845 West Taylor Street, Chicago, Illinois 60607 USA

\section{Supporting Information}

\section{Table of Contents}

I. General Information S-2

II. General Procedures S-3

III. Experimental Data for Coupling Products (Tables 2 \& 3, Eq. 1) S-3

IV. Procedures and Data for Synthetic Applications S-17

$\begin{array}{lll}\text { V. References } & \text { S-19 }\end{array}$

VI. NMR Spectra for Cycloadducts S-20 


\section{General Information}

Unless otherwise stated, reactions were performed in oven-dried glasswares under a $\mathrm{N}_{2}$ atmosphere using dry, deoxygenated solvents. Anhydrous dichloromethane, pentane, toluene, THF, and acetonitrile were purchased from VWR or Fisher, degassed with argon, and dried by passage through activated drying columns ${ }^{1}$ on a Pure Process Technology system. Cyclohexandione and other dicarbonyls were purchased from Aldrich, Alfa, or Oakwood and were used as received. $\mathrm{Mn}(\mathrm{OAc})_{3} \cdot 2 \mathrm{H}_{2} \mathrm{O}$ was prepared according to the literature procedure. ${ }^{2}$ The TBS dienol ethers were synthesized by enolization with LiHMDS or by soft enolization according to our previous publication. ${ }^{3}$ Deuterated chloroform $\left(\mathrm{CDCl}_{3}, 99.9 \%\right.$, extra dry) was purchased from Cambridge Isotope Laboratories, Inc. and was used without further purification. Reaction temperatures were controlled by an IKAmag immersion temperature modulator. Thin-layer chromatography (TLC) was performed using Silicycle silica gel 60 F254 precoated plates $(0.25$ $\mathrm{mm}$ ) and visualized by UV fluorescence quenching or staining with $p$-anisaldehyde or $\mathrm{KMnO}_{4}$ solutions. Flash chromatography ${ }^{4}$ was performed using Silicycle SiliaFlashR P60 silica gel (40$63 \mu \mathrm{m}$ particle size). Melting points were determined using a Mel-Temp electrothermal capillary melting point apparatus and the values reported are uncorrected. ${ }^{1} \mathrm{H}$ and ${ }^{13} \mathrm{C}$ NMR spectra were recorded on a Bruker Avance DRX-500 (at 500 and $126 \mathrm{MHz}$, respectively) or DPX-400 instrument (at 400 and $101 \mathrm{MHz}$, respectively) and are reported relative to $\mathrm{Me}_{4} \mathrm{Si}(\delta 0.0)$. Data for ${ }^{1} \mathrm{H}$ NMR spectra are reported as follows: chemical shift ( $\left.\delta \mathrm{ppm}\right)$ (multiplicity, coupling constant $(\mathrm{Hz})$, integration). Multiplicities are reported as follows: $\mathrm{s}=$ singlet, $\mathrm{d}=$ doublet, $\mathrm{t}=$ triplet, $\mathrm{q}=$ quartet, sept $=$ septet, $\mathrm{m}=$ multiplet, comp. $\mathrm{m}=$ complex multiplet, app. = apparent, br $\mathrm{s}=$ broad singlet. Data for ${ }^{13} \mathrm{C}$ NMR spectra are reported in terms of chemical shift relative to $\mathrm{Me}_{4} \mathrm{Si}(\delta 0.0)$. Infrared (IR) spectra were recorded on a Nicolet iS5 FTIR spectrometer and are reported in frequency of absorption $\left(\mathrm{cm}^{-1}\right)$. GC/MS analyses were performed on a Hewlett Packard Model 6890 gas chromatograph interfaced to a Hewlett Packard Model 5973 mass selective detector (15 m x $0.25 \mathrm{~mm}$ capillary column, HP-5MS). 


\section{General Procedures}

The TBS dienol ethers were synthesized by enolization with LiHMDS or by soft enolization according to our previously published methods. ${ }^{3}$

\section{General Procedure for Mn-Mediated $\gamma$-Alkylation of Enones with 1,3-Dicarbonyls}

An oven-dried $20 \mathrm{~mL}$ vial equipped with a magnetic stirring bar was charged with $\mathrm{Mn}(\mathrm{OAc})_{3} \cdot 2 \mathrm{H}_{2} \mathrm{O}$ (150.8 $\mathrm{mg}, 0.56 \mathrm{mmol}, 2.25$ equiv) and 1,3-dicarbonyl compound ( $0.275 \mathrm{mmol}, 1.1$ equiv) if the dicarbonyl was a solid. The vial was sealed with a septum cap, evacuated, and refilled with $\mathrm{N}_{2}$ (three cycles). Anhydrous acetonitrile $(7.5 \mathrm{~mL}$ ) and TBS dienol ether $(0.25 \mathrm{mmol}, 1.0$ equiv) were injected into the vial sequentially (followed by neat 1,3-dicarbonyl compound, if it was a liquid). The mixture was then heated to $80{ }^{\circ} \mathrm{C}$ for $2-4 \mathrm{~h}$. After the reaction was complete (monitored by TLC), the mixture was cooled to room temperature and filtered through a pad of Celite. After concentration of the filtrate by rotary evaporation, the residue was purified by flash chromatography on silica gel to afford the desired product.

Specific quantities of reagents, procedural variations, and purification conditions may be found below in the entry containing the characterization data.

\section{Experimental Data for $\gamma-\mathrm{C}-\mathrm{C}$ Coupling Products (Tables 1, 2 \& 3, Eqs. 2,3 \& 4)}

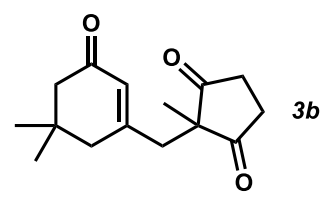

2-((5,5-Dimethyl-3-oxocyclohex-1-en-1-yl)methyl)-2-methylcyclopentane-1,3-dione (3b): The title compound was prepared according to the general procedure using crude tert-butyl $((5,5-$ dimethyl-3-methylenecyclohex-1-en-1-yl)oxy)dimethylsilane (1a, prepared according to ref 3) (63.0 mg, $0.25 \mathrm{mmol}, 1.0$ equiv) and 2-methylcyclopentane-1,3-dione (2b, $30.8 \mathrm{mg}, 0.275 \mathrm{mmol}$, 1.1 equiv) at $80{ }^{\circ} \mathrm{C}$ for $2 \mathrm{~h}$. After purification by flash chromatography $\left(\mathrm{SiO}_{2}\right.$, hexanes $/ \mathrm{EtOAc}=$ $2: 1)$, the title compound was isolated as a white solid.

$1^{\text {st }}$ run: $44.6 \mathrm{mg}(72 \%$ yield $)$

$2^{\text {nd }}$ run: $42.2 \mathrm{mg}(68 \%$ yield $)$

TLC $\left(\mathrm{SiO}_{2}\right) R_{f}=0.30$ in 1:1 hexanes/EtOAc, $p$-anisaldehyde stain

mp $72-73{ }^{\circ} \mathrm{C}$

${ }^{1} \mathbf{H}$ NMR $\left(500 \mathrm{MHz}, \mathrm{CDCl}_{3}\right) \delta 5.53(\mathrm{~s}, 1 \mathrm{H}), 2.86(\mathrm{~m}, 2 \mathrm{H}), 2.71(\mathrm{~m}, 2 \mathrm{H}), 2.55(\mathrm{~s}, 2 \mathrm{H}), 2.11(\mathrm{~s}$, $2 \mathrm{H}), 2.07(\mathrm{~s}, 2 \mathrm{H}), 1.16(\mathrm{~s}, 3 \mathrm{H}), 0.95(\mathrm{~s}, 6 \mathrm{H})$

${ }^{13}$ C NMR $\left(126 \mathrm{MHz}, \mathrm{CDCl}_{3}\right) \delta 215.0,199.2,158.0,127.3,56.4,50.7,45.3,41.3,35.0,33.6$, $28.0,22.0$

IR (neat) 2957, 2926, 1764, 1722, 1666, 1451, 1299, $1065 \mathrm{~cm}^{-1}$

HRMS $\left(\mathrm{EI}^{+}\right) \mathrm{m} / z$ calc'd for $\mathrm{C}_{15} \mathrm{H}_{20} \mathrm{O}_{3}[\mathrm{M}]^{+}: 248.1419$, found 248.1413 


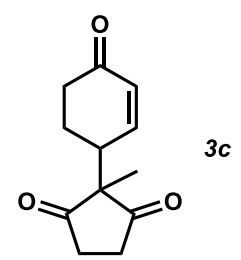

2-Methyl-2-(4-oxocyclohex-2-en-1-yl)cyclopentane-1,3-dione (3c): The title compound was prepared according to the General Procedure, using tert-butyl(cyclohexa-1,3-dien-1yloxy)dimethylsilane (1b, $52.5 \mathrm{mg}, 0.25 \mathrm{mmol}, 1.0$ equiv) and 2-methylcyclopentane-1,3-dione (2b, $30.8 \mathrm{mg}, 0.275 \mathrm{mmol}, 1.1$ equiv) at $80{ }^{\circ} \mathrm{C}$ for $2 \mathrm{~h}$. After purification by flash chromatography $\left(\mathrm{SiO}_{2}\right.$, hexanes/EtOAc $\left.=1: 1\right)$, the title compound was isolated as a white solid.

$1^{\text {st }}$ run: $40.2 \mathrm{mg}$ (78\% yield)

$2^{\text {nd }}$ run: $38.1 \mathrm{mg}(74 \%$ yield $)$

TLC $\left(\mathrm{SiO}_{2}\right) R_{f}=0.25$ in 1:2 hexanes/EtOAc, $p$-anisaldehyde stain

mp $75-76^{\circ} \mathrm{C}$

${ }^{1}$ H NMR $\left(500 \mathrm{MHz}, \mathrm{CDCl}_{3}\right) \delta 6.90(\mathrm{~d}, J=10.4 \mathrm{~Hz}, 1 \mathrm{H}), 6.06(\mathrm{dd}, 1 \mathrm{H}, J=10.4,1.9 \mathrm{~Hz}, 1 \mathrm{H})$, 2.81 (comp. m, 5H), $2.48(\mathrm{~m}, 1 \mathrm{H}), 2.31(\mathrm{~m}, 1 \mathrm{H}), 1.94$ (br. s, 1H), $1.79(\mathrm{~m}, 1 \mathrm{H}), 1.22(\mathrm{~s}, 3 \mathrm{H})$

${ }^{13}$ C NMR (126 MHz, $\left.\mathrm{CDCl}_{3}\right) \delta$ 214.9, 214.8, 198.1, 148.1, 130.9, 58.0, 40.8, 37.2, 35.5, 35.4, $25.0,17.2$

IR (neat) 2950, 2933, 1717, 1674, 1451, $1082 \mathrm{~cm}^{-1}$

HRMS $\left(\mathrm{EI}^{+}\right) \mathrm{m} / z$ calc'd for $\mathrm{C}_{12} \mathrm{H}_{15} \mathrm{O}_{3}[\mathrm{M}]^{+}:$: 207.1022, found 207.1021

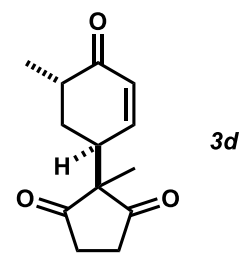

2-Methyl-2-(5-methyl-4-oxocyclohex-2-en-1-yl)cyclopentane-1,3-dione $\quad(3 \mathrm{~d}): \quad$ The title compound was prepared according to the General Procedure, using tert-butyldimethyl((6methylcyclohexa-1,3-dien-1-yl)oxy)silane (1c, $56.0 \mathrm{mg}, 0.25 \mathrm{mmol}, 1.0$ equiv) and 2methylcyclopentane-1,3-dione (2b, $30.8 \mathrm{mg}, 0.275 \mathrm{mmol}, 1.1$ equiv) at $80{ }^{\circ} \mathrm{C}$ for $2 \mathrm{~h}$. Crude ${ }^{1} \mathrm{H}$ NMR prior to purification indicated a 6.6:1 mixture of diastereomers. After purification by flash chromatography $\left(\mathrm{SiO}_{2}\right.$, hexanes/EtOAc $\left.=1: 1\right)$, the title compound was isolated as a white solid, comprising a 6.6:1 mixture of diastereomers (based on ${ }^{1} \mathrm{H}$ NMR of purified material).

$1^{\text {st }}$ run: $38.5 \mathrm{mg}(70 \%$ yield $)$

$2^{\text {nd }}$ run: $40.7 \mathrm{mg}(74 \%$ yield $)$

TLC $\left(\mathrm{SiO}_{2}\right) R_{f}=0.35$ in 1:2 hexanes/EtOAc, $p$-anisaldehyde stain

mp $109-110^{\circ} \mathrm{C}$

${ }^{1} \mathbf{H}$ NMR $\left(500 \mathrm{MHz}, \mathrm{CDCl}_{3}\right) \delta 6.81(\mathrm{~d}, J=10.4 \mathrm{~Hz}, 1 \mathrm{H}), 5.99(\mathrm{dd}, J=10.4,2.6 \mathrm{~Hz}, 1 \mathrm{H}), 2.83$ (comp. m, 5H), 2.52 (m, 1H), $1.98(\mathrm{~m}, 1 \mathrm{H}), 1.73(\mathrm{~m}, 1 \mathrm{H}), 1.22$ (s, 3H), 1.12 (d, J= $7.3 \mathrm{~Hz}$, $3 \mathrm{H})$

${ }^{13}$ C NMR (126 MHz, $\left.\mathrm{CDCl}_{3}\right) \delta$ 215.1, 215.0, 201.4, 146.7, 129.7, 57.9, 39.4, 37.3, 35.5, 31.0, $17.4,15.5$

IR (neat) 2967, 2933, 2873, 1762, 1717, 1673, 1450, 1291, $1083 \mathrm{~cm}^{-1}$

HRMS $\left(\mathrm{EI}^{+}\right) \mathrm{m} / z$ calc'd for $\mathrm{C}_{13} \mathrm{H}_{16} \mathrm{O}_{3}[\mathrm{M}]^{+}: 220.1093$, found 220.1100 


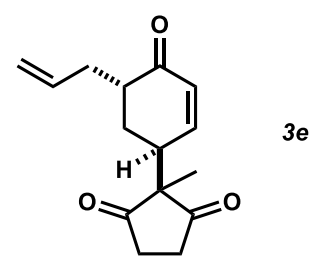

2-Methyl-2-(5-methyl-4-oxocyclohex-2-en-1-yl)cyclopentane-1,3-dione (3e): The title compound was prepared according to the General Procedure, using ((6-allylcyclohexa-1,3-dien1-yl)oxy)(tert-butyl)dimethylsilane $(\mathbf{1 d}, \quad 62.5 \mathrm{mg}, \quad 0.25 \mathrm{mmol}, \quad 1.0$ equiv) and 2methylcyclopentane-1,3-dione (2b, $30.8 \mathrm{mg}, 0.275 \mathrm{mmol}, 1.1$ equiv) at $80{ }^{\circ} \mathrm{C}$ for $2 \mathrm{~h}$. Crude ${ }^{1} \mathrm{H}$ NMR prior to purification indicated a 6.2:1 mixture of diastereomers. After purification by flash chromatography $\left(\mathrm{SiO}_{2}\right.$, hexanes/EtOAc $\left.=1: 1\right)$, the title compound was isolated as a colorless oil, comprising a 6.2:1 mixture of diastereomers (based on ${ }^{1} \mathrm{H}$ NMR of purified material).

$1^{\text {st }}$ run: $35.7 \mathrm{mg}(58 \%$ yield $)$

$2^{\text {nd }}$ run: $36.9 \mathrm{mg}(60 \%$ yield $)$

TLC $\left(\mathrm{SiO}_{2}\right) R_{f}=0.35$ in 1:2 hexanes/EtOAc, $p$-anisaldehyde stain

${ }^{1} \mathbf{H}$ NMR $\left(500 \mathrm{MHz}, \mathrm{CDCl}_{3}\right) \delta 6.81(\mathrm{~d}, J=10.5 \mathrm{~Hz}, 1 \mathrm{H}), 6.00(\mathrm{dd}, J=10.5,2.5 \mathrm{~Hz}, 1 \mathrm{H}), 5.69$ (ddd, $J=15.6,9.3,6.2 \mathrm{~Hz}, 1 \mathrm{H}), 5.09$ (d, $J=4.4 \mathrm{~Hz}, 1 \mathrm{H}), 5.06$ (s, 1H), 2.82 (comp. m, 5H), $2.43(\mathrm{~m}, 1 \mathrm{H}), 2.34(\mathrm{~m}, 1 \mathrm{H}), 2.14(\mathrm{~m}, 1 \mathrm{H}), 1.91(\mathrm{~m}, 1 \mathrm{H}), 1.84(\mathrm{~m}, 1 \mathrm{H}), 1.20(\mathrm{~s}, 3 \mathrm{H})$

${ }^{13}$ C NMR $\left(126 \mathrm{MHz}, \mathrm{CDCl}_{3}\right) \delta$ 215.0, 214.8, 200.1, 146.9, 135.1, 129.9, 117.7, 57.9, 44.4, 37.1, $35.5,33.9,27.7,17.3$

IR (neat) 2931, 1719, 1673, 1448, $918 \mathrm{~cm}^{-1}$

HRMS $\left(\mathrm{EI}^{+}\right) \mathrm{m} / z$ calc'd for $\mathrm{C}_{15} \mathrm{H}_{18} \mathrm{O}_{3}[\mathrm{M}]^{+}:$: 246.1249, found 246.1256

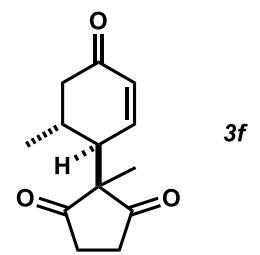

2-Methyl-2-(6-methyl-4-oxocyclohex-2-en-1-yl)cyclopentane-1,3-dione (3f): The title compound was prepared according to the General Procedure, using tert-butyldimethyl $((5-$ methylcyclohexa-1,3-dien-1-yl)oxy)silane (1e, $56.0 \mathrm{mg}, 0.25 \mathrm{mmol}, 1.0$ equiv) and 2methylcyclopentane-1,3-dione (2b, $30.8 \mathrm{mg}, 0.275 \mathrm{mmol}, 1.1$ equiv) at $80{ }^{\circ} \mathrm{C}$ for $2 \mathrm{~h}$. Crude ${ }^{1} \mathrm{H}$ NMR prior to purification indicated a $>20: 1$ mixture of diastereomers. After purification by flash chromatography $\left(\mathrm{SiO}_{2}\right.$, hexanes/EtOAc $\left.=1: 1\right)$, the title compound was isolated as a colorless oil, comprising a $>20: 1$ mixture of diastereomers (based on ${ }^{1} \mathrm{H}$ NMR of purified material).

$1^{\text {st }}$ run: $29.7 \mathrm{mg}(54 \%$ yield $)$

$2^{\text {nd }}$ run: $28.6 \mathrm{mg}(52 \%$ yield $)$

TLC $\left(\mathrm{SiO}_{2}\right) R_{f}=0.40$ in 1:2 hexanes/EtOAc, $p$-anisaldehyde stain

${ }^{1} \mathbf{H}$ NMR $\left(400 \mathrm{MHz}, \mathrm{CDCl}_{3}\right) \delta 6.76(\mathrm{dd}, J=10.4,3.6 \mathrm{~Hz}, 1 \mathrm{H}), 6.10(\mathrm{dd}, J=10.4,2.1 \mathrm{~Hz}, 1 \mathrm{H})$, 2.86 (comp. m, 4H), 2.61 (dd, $J=6.1,4.0 \mathrm{~Hz}, 1 \mathrm{H}), 2.55$ (dd, $J=16.7,5.0 \mathrm{~Hz}, 1 \mathrm{H}), 2.25$ (m, $1 \mathrm{H}), 2.14(\mathrm{dd}, J=16.7,5.9 \mathrm{~Hz}, 1 \mathrm{H}), 1.25(\mathrm{~s}, 3 \mathrm{H}), 0.96(\mathrm{~d}, J=7.0 \mathrm{~Hz}, 3 \mathrm{H})$

${ }^{13}$ C NMR (101 MHz, $\left.\mathrm{CDCl}_{3}\right) \delta$ 215.1, 214.8, 198.0, 145.7, 130.8, 58.9, 46.2, 43.1, 35.3, 35.2, $30.3,22.2,19.0$

IR (neat) 2962, 1756, 1717, 1673, 1455, $1079 \mathrm{~cm}^{-1}$ 
HRMS $\left(\mathrm{EI}^{+}\right) \mathrm{m} / z$ calc'd for $\mathrm{C}_{13} \mathrm{H}_{17} \mathrm{O}_{3}\left[\mathrm{M}^{+}: 221.1177\right.$, found 221.1178

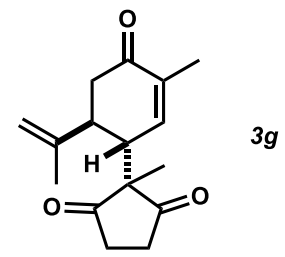

2-Methyl-2-((1S,6S)-3-methyl-4-oxo-6-(prop-1-en-2-yl)cyclohex-2-en-1-yl)cyclopentane-1,3dione (3g): The title compound was prepared according to the General Procedure, using $(S)$-tertbutyldimethyl((2-methyl-5-(prop-1-en-2-yl)cyclohexa-1,3-dien-1-yl)oxy)silane (1f, $66.0 \mathrm{mg}$, $0.25 \mathrm{mmol}, 1.0$ equiv) and 2-methylcyclopentane-1,3-dione (2b, $30.8 \mathrm{mg}, 0.275 \mathrm{mmol}, 1.1$ equiv) at $80{ }^{\circ} \mathrm{C}$ for $2 \mathrm{~h}$. Crude ${ }^{1} \mathrm{H}$ NMR prior to purification indicated a single diastereomer. After purification by flash chromatography $\left(\mathrm{SiO}_{2}\right.$, hexanes/EtOAc $\left.=1: 1\right)$, the title compound was isolated as a colorless oil as a single diastereomer (based on ${ }^{1} \mathrm{H}$ NMR of purified material).

$1^{\text {st }}$ run: $22.8 \mathrm{mg}(35 \%$ yield $)$

$2^{\text {nd }}$ run: $22.8 \mathrm{mg}(35 \%$ yield $)$

TLC $\left(\mathrm{SiO}_{2}\right) R_{f}=0.45$ in 1:2 hexanes/EtOAc, $p$-anisaldehyde stain

${ }^{1}$ H NMR $\left(500 \mathrm{MHz}, \mathrm{CDCl}_{3}\right) \delta 6.78(\mathrm{~s}, 1 \mathrm{H}), 4.71(\mathrm{~s}, 1 \mathrm{H}), 4.69(\mathrm{~s}, 1 \mathrm{H}), 3.03(\mathrm{~d}, J=9.5 \mathrm{~Hz}, 1 \mathrm{H})$, 2.76 (comp. m, 5H), 2.42 (dd, $J=16.1,12.4 \mathrm{~Hz}, 1 \mathrm{H}), 2.33$ (dd, $J=16.1,4.1 \mathrm{~Hz}, 1 \mathrm{H}), 1.83$ $(\mathrm{s}, 3 \mathrm{H}), 1.57(\mathrm{~s}, 3 \mathrm{H}), 1.27(\mathrm{~s}, 3 \mathrm{H})$

${ }^{13}$ C NMR $\left(126 \mathrm{MHz}, \mathrm{CDCl}_{3}\right) \delta$ 215.6, 213.0, 198.4, 146.3, 143.3, 136.2, 115.5, 58.4, 46.6, 42.6, $42.4,34.8,34.1,21.1,18.3,16.2$

IR (neat) $3100,2923,1718,1671,1447,1111 \mathrm{~cm}^{-1}$

HRMS $\left(\mathrm{EI}^{+}\right) \mathrm{m} / \mathrm{z}$ calc'd for $\mathrm{C}_{12} \mathrm{H}_{21} \mathrm{O}_{6}[\mathrm{M}]^{+}: 261.1342$, found 261.1338

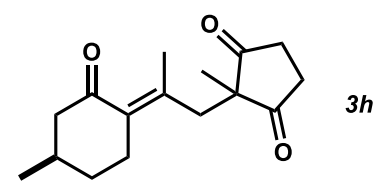

(R)-2-Methyl-2-(2-(4-methyl-2-oxocyclohexylidene)propyl)cyclopentane-1,3-dione (3h): The title compound was prepared according to the General Procedure, using $(R)$-tertbutyldimethyl((5-methyl-2-(prop-1-en-2-yl)cyclohex-1-en-1-yl)oxy)silane (1g, $66.5 \mathrm{mg}, 0.25$ mmol, 1.0 equiv) and 2-methylcyclopentane-1,3-dione (2b, $30.8 \mathrm{mg}, 0.275 \mathrm{mmol}, 1.1$ equiv) at $80{ }^{\circ} \mathrm{C}$ for $2 \mathrm{~h}$. After purification by flash chromatography $\left(\mathrm{SiO}_{2}\right.$, hexanes/EtOAc $\left.=1: 1\right)$, the title compound was isolated as a colorless liquid. Partial separation was possible allowing the characterization of the individual stereoisomers.

\section{E-isomer}

$1^{\text {st }}$ run: $55.7 \mathrm{mg}(85 \%$ yield $)$

$2^{\text {nd }}$ run: $57.0 \mathrm{mg}(87 \%$ yield $)$

TLC $\left(\mathrm{SiO}_{2}\right) R_{f}=0.45$ in 1:2 hexanes/EtOAc, $p$-anisaldehyde stain

${ }^{1} \mathbf{H}$ NMR $\left(500 \mathrm{MHz}, \mathrm{CDCl}_{3}\right) \delta 3.01(\mathrm{~m}, 2 \mathrm{H}), 2.65(\mathrm{~m}, 5 \mathrm{H}), 2.42(\mathrm{~d}, J=11.6 \mathrm{~Hz}, 1 \mathrm{H}), 2.26(\mathrm{~m}$, $1 \mathrm{H}), 1.94(\mathrm{~m}, 2 \mathrm{H}), 1.86(\mathrm{~s}, 3 \mathrm{H}), 1.30(\mathrm{~m}, 1 \mathrm{H}), 1.16(\mathrm{~s}, 3 \mathrm{H}), 0.98(\mathrm{~d}, J=6.1 \mathrm{~Hz}, 3 \mathrm{H})$

${ }^{13}$ C NMR (126 MHz, $\left.\mathrm{CDCl}_{3}\right) \delta$ 216.2, 215.6, 203.7, 141.9, 133.9, 55.9, 50.5, 42.3, 35.2, 35.0, $32.2,30.8,28.6,23.3,21.6,19.8$

IR (neat) 2950, 2926, 2870, 1721, 1674, 1455, $1282 \mathrm{~cm}^{-1}$

HRMS $\left(\mathrm{EI}^{+}\right) \mathrm{m} / z$ calc'd for $\mathrm{C}_{16} \mathrm{H}_{22} \mathrm{O}_{3}[\mathrm{M}]^{+}: 262.1574$, found 262.1569 


\section{$Z$-isomer}

TLC $\left(\mathrm{SiO}_{2}\right) R_{f}=0.40$ in 1:2 hexanes/EtOAc, $p$-anisaldehyde stain

${ }^{1}$ H NMR (500 MHz, $\left.\mathrm{CDCl}_{3}\right) \delta 2.75$ (comp. m, 5H), 2.63 (d, $\left.J=13.1 \mathrm{~Hz}, 1 \mathrm{H}\right), 2.46(\mathrm{~m}, 2 \mathrm{H}), 2.12$ $(\mathrm{m}, 1 \mathrm{H}), 1.98(\mathrm{~m}, 2 \mathrm{H}), 1.86$ (app. s, $1 \mathrm{H}), 1.68(\mathrm{~s}, 3 \mathrm{H}), 1.31(\mathrm{~m}, 1 \mathrm{H}), 1.15(\mathrm{~s}, 3 \mathrm{H}), 0.99$ (d, $J$ $=6.1 \mathrm{~Hz}, 3 \mathrm{H})$

${ }^{13}$ C NMR (126 MHz, $\left.\mathrm{CDCl}_{3}\right) \delta$ 216.5, 216.3, 205.6, 137.6, 134.7, 56.2, 51.7, 41.4, 35.8, 35.7, $34.8,33.5,32.7,29.5,21.8,20.6$

IR (neat) 2950, 2926, 2870, 1720, 1684, 1454, 1418, $1072 \mathrm{~cm}^{-1}$

HRMS $\left(\mathrm{EI}^{+}\right) \mathrm{m} / z$ calc'd for $\mathrm{C}_{16} \mathrm{H}_{22} \mathrm{O}_{3}[\mathrm{M}]^{+}: 262.1562$, found 262.1569

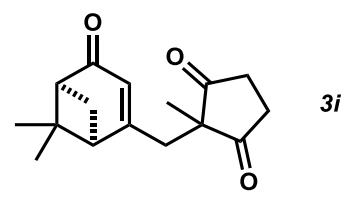

2-(((1R,5S)-6,6-Dimethyl-4-oxobicyclo[3.1.1] hept-2-en-2-yl)methyl)-2-methylcyclopentane1,3-dione (3i): The title compound was prepared according to the general procedure using crude tert-butyl(((1S,5R)-6,6-dimethyl-4-methylenebicyclo[3.1.1]hept-2-en-2-yl)oxy)dimethylsilane (1h, prepared according to ref 3) $(66.0 \mathrm{mg}, 0.25 \mathrm{mmol}, 1.0$ equiv) and 2-methylcyclopentane1,3-dione (2a, $30.8 \mathrm{mg}, 0.275 \mathrm{mmol}, 1.1$ equiv) at $80{ }^{\circ} \mathrm{C}$ for $2 \mathrm{~h}$. After purification by flash chromatography $\left(\mathrm{SiO}_{2}\right.$, hexanes/EtOAc $\left.=2: 1\right)$, the title compound was isolated as a white solid.

$1^{\text {st }}$ run: $44.2 \mathrm{mg}(68 \%$ yield $)$

$2^{\text {nd }}$ run: $45.5 \mathrm{mg}(70 \%$ yield $)$

TLC $\left(\mathrm{SiO}_{2}\right) R_{f}=0.35$ in 1:1 hexanes/EtOAc, $p$-anisaldehyde stain

mp $106-107^{\circ} \mathrm{C}$

${ }^{1} \mathbf{H}$ NMR $\left(500 \mathrm{MHz}, \mathrm{CDCl}_{3}\right) \delta 5.31(\mathrm{~s}, 1 \mathrm{H}), 2.88(\mathrm{~m}, 2 \mathrm{H}), 2.74(\mathrm{~m}, 3 \mathrm{H}), 2.63(\mathrm{~d}, J=3.8 \mathrm{~Hz}, 2 \mathrm{H})$, $2.57(\mathrm{t}, J=5.3 \mathrm{~Hz}, 1 \mathrm{H}), 2.34(\mathrm{t}, J=5.3 \mathrm{~Hz}, 1 \mathrm{H}), 1.93(\mathrm{~d}, J=9.3 \mathrm{~Hz}, 1 \mathrm{H}), 1.43(\mathrm{~s}, 3 \mathrm{H})$, $1.16(\mathrm{~s}, 3 \mathrm{H}), 0.90(\mathrm{~s}, 3 \mathrm{H})$

${ }^{13}$ C NMR (126 MHz, $\left.\mathrm{CDCl}_{3}\right) \delta$ 214.8, 214.5, 203.0, 167.4, 122.9, 57.5, 56.4, 54.1, 49.7, 40.8, $40.5,34.8,34.7,26.5,22.1,21.9$

IR (neat) 2957, 1763, 1721, 1671, 1283, $1062 \mathrm{~cm}^{-1}$

HRMS $\left(\mathrm{EI}^{+}\right) \mathrm{m} / z$ calc'd for $\mathrm{C}_{16} \mathrm{H}_{20} \mathrm{O}_{3}[\mathrm{M}]^{+}: 260.1417$, found 260.1413

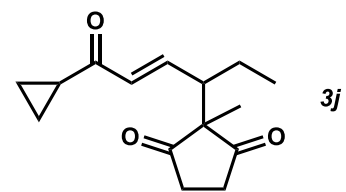

(E)-2-(6-Cyclopropyl-6-oxohex-4-en-3-yl)-2-methylcyclopentane-1,3-dione (3j): The title compound was prepared according to the general procedure using crude tert-butyl $(((1 Z, 3 E)-1$ cyclopropylhexa-1,3-dien-1-yl)oxy)dimethylsilane (1i, prepared according to ref 3) (63.0 mg, $0.25 \mathrm{mmol}, 1.0$ equiv) and 2-methylcyclopentane-1,3-dione (2b, $30.8 \mathrm{mg}, 0.275 \mathrm{mmol}, 1.1$ equiv) at $80{ }^{\circ} \mathrm{C}$ for $2 \mathrm{~h}$. After purification by flash chromatography $\left(\mathrm{SiO}_{2}\right.$, hexanes/EtOAc $\left.=2: 1\right)$, the title compound was isolated as a colorless liquid.

$1^{\text {st }}$ run: $39.1 \mathrm{mg}(63 \%$ yield $)$

$2^{\text {nd }}$ run: $37.8 \mathrm{mg}(61 \%$ yield $)$ 
TLC $\left(\mathrm{SiO}_{2}\right) R_{f}=0.35$ in $1: 1$ hexanes/EtOAc, $p$-anisaldehyde stain

${ }^{1}$ H NMR $\left(400 \mathrm{MHz}, \mathrm{CDCl}_{3}\right) \delta 6.78(\mathrm{dd}, J=15.8,10.5 \mathrm{~Hz}, 1 \mathrm{H}), 6.17(\mathrm{~d}, J=15.8 \mathrm{~Hz}, 1 \mathrm{H}), 2.79$ $(\mathrm{m}, 4 \mathrm{H}), 2.41(\mathrm{dt}, J=10.7,3.3 \mathrm{~Hz}, 1 \mathrm{H}), 2.18(\mathrm{~m}, 1 \mathrm{H}), 1.38(\mathrm{~m}, 2 \mathrm{H}), 1.09$ (app. s, 5H), 0.93 $(\mathrm{m}, 2 \mathrm{H}), 0.78(\mathrm{t}, J=7.3 \mathrm{~Hz}, 3 \mathrm{H})$

${ }^{13}$ C NMR (101 MHz, $\left.\mathrm{CDCl}_{3}\right) \delta 216.1,199.7,143.4,134.2,58.7,50.3,35.7,35.6,22.8,19.5$, $18.5,12.5,11.4,11.3$

IR (neat) 2966, 2930, 2876, 1717, 1682, 1656, 1418, 1087, $1047 \mathrm{~cm}^{-1}$

HRMS $\left(\mathrm{EI}^{+}\right) \mathrm{m} / z$ calc'd for $\mathrm{C}_{15} \mathrm{H}_{20} \mathrm{O}_{3}[\mathrm{M}]^{+}:$:248.1414, found 248.1413<smiles>CC12CCCC(C3(C)C(=O)CCC3=O)C1=CC(=O)CC2</smiles>

2-methyl-2-(4a-methyl-7-oxo-1,2,3,4,4a,5,6,7-octahydronaphthalen-1-yl)cyclopentane-1,3dione (3k): The title compound was prepared according to the general procedure using crude tert-butyldimethyl((4a-methyl-3,4,4a,5,6,7-hexahydronaphthalen-2-yl)oxy)silane (1jj, prepared according to ref 3) $(69.5 \mathrm{mg}, 0.25 \mathrm{mmol}, 1.0$ equiv) and 2-methylcyclopentane-1,3-dione (2b, $30.8 \mathrm{mg}, 0.275 \mathrm{mmol}, 1.1$ equiv) at $80{ }^{\circ} \mathrm{C}$ for $2 \mathrm{~h}$. Crude ${ }^{1} \mathrm{H}$ NMR prior to purification indicated a single diastereomer. After purification by flash chromatography $\left(\mathrm{SiO}_{2}\right.$, hexanes/EtOAc $\left.=2: 1\right)$, the title compound was isolated as a colorless oil as a single diastereomer (based on ${ }^{1} \mathrm{H}$ NMR of purified material).

$1^{\text {st }}$ run: $30.1 \mathrm{mg}(44 \%$ yield $)$

$2^{\text {nd }}$ run: $31.5 \mathrm{mg}(46 \%$ yield $)$

TLC $\left(\mathrm{SiO}_{2}\right) R_{f}=0.35$ in $1: 1$ hexanes/EtOAc, $p$-anisaldehyde stain

${ }^{1}$ H NMR $\left(500 \mathrm{MHz}, \mathrm{CDCl}_{3}\right) \delta 5.62(\mathrm{~s}, 1 \mathrm{H}), 3.34(\mathrm{dd}, J=15.0,4.4 \mathrm{~Hz}, 1 \mathrm{H}), 3.09(\mathrm{~m}, 1 \mathrm{H}), 2.83$ $(\mathrm{m}, 2 \mathrm{H}), 2.71(\mathrm{~m}, 1 \mathrm{H}), 2.31(\mathrm{~m}, 3 \mathrm{H}), 1.88$ (br. s, $1 \mathrm{H}), 1.81(\mathrm{dd}, J=13.0,4.5 \mathrm{~Hz}, 1 \mathrm{H}), 1.68$ $(\mathrm{m}, 4 \mathrm{H}), 1.43(\mathrm{~m}, 2 \mathrm{H}), 1.30(\mathrm{~s}, 3 \mathrm{H}), 1.05(\mathrm{~s}, 3 \mathrm{H})$

${ }^{13}$ C NMR $\left(126 \mathrm{MHz}, \mathrm{CDCl}_{3}\right) \delta$ 218.0, 216.1, 199.0, 172.0, 122.6, 53.1, 52.0, 42.0, 38.6, 37.2, $34.9,34.2,32.7,27.1,21.8,21.7,17.4$

IR (neat) 2932, 2863, 1718, 1658, 1370, $1228 \mathrm{~cm}^{-1}$

HRMS $\left(\mathrm{EI}^{+}\right) m / z$ calc'd for $\mathrm{C}_{17} \mathrm{H}_{22} \mathrm{O}_{3}[\mathrm{M}]^{+}:$: 274.1569, found 274.1569<smiles>CC1(C2C=CC(=O)CC2)C(=O)CCCC1=O</smiles>

1-Methyl-[1,1'-bi(cyclohexan)]-2'-ene-2,4',6-trione (3I): The title compound was prepared according to the General Procedure, using tert-butyl(cyclohexa-1,3-dien-1-yloxy)dimethylsilane (1b, $52.5 \mathrm{mg}, 0.25 \mathrm{mmol}, 1.0$ equiv) and 2-methylcyclohexane-1,3-dione (2c, $34.6 \mathrm{mg}, 0.275$ mmol, 1.1 equiv) at $80{ }^{\circ} \mathrm{C}$ for $2 \mathrm{~h}$. After purification by flash chromatography $\left(\mathrm{SiO}_{2}\right.$, hexanes/EtOAc $=1: 1)$, the title compound was isolated as a colorless liquid.

$1^{\text {st }}$ run: $43.5 \mathrm{mg}(79 \%$ yield $)$

$2^{\text {nd }}$ run: $44.5 \mathrm{mg}(81 \%$ yield $)$ 
TLC $\left(\mathrm{SiO}_{2}\right) R_{f}=0.25$ in 1:2 hexanes/EtOAc, $p$-anisaldehyde stain

${ }^{1} \mathbf{H}$ NMR $\left(500 \mathrm{MHz}, \mathrm{CDCl}_{3}\right) \delta 6.73(\mathrm{~d}, J=10.3 \mathrm{~Hz}, 1 \mathrm{H}), 6.01(\mathrm{dd}, J=10.3,1.9 \mathrm{~Hz}, 1 \mathrm{H}), 3.19$ $(\mathrm{m}, 1 \mathrm{H}), 2.69(\mathrm{~m}, 4 \mathrm{H}), 2.48(\mathrm{~d}, J=16.6 \mathrm{~Hz}, 1 \mathrm{H}), 2.34(\mathrm{~m}, 1 \mathrm{H}), 2.03(\mathrm{~m}, 1 \mathrm{H}), 1.89(\mathrm{~m}, 1 \mathrm{H})$, 1.80 (br. s, $2 \mathrm{H}), 1.25$ (s, 3H)

${ }^{13}$ C NMR (126 MHz, $\left.\mathrm{CDCl}_{3}\right) \delta$ 209.0, 208.4, 198.2, 149.2, 130.0, 67.9, 41.5, 38.3, 37.9, 37.5, $25.2,17.6,16.3$

IR (neat) 2952, 1723, 1689, 1425, 1319, 1072, $841 \mathrm{~cm}^{-1}$

HRMS $\left(\mathrm{EI}^{+}\right) \mathrm{m} / z$ calc'd for $\mathrm{C}_{13} \mathrm{H}_{16} \mathrm{O}_{3}[\mathrm{M}]^{+}: 220.1093$, found 220.1100

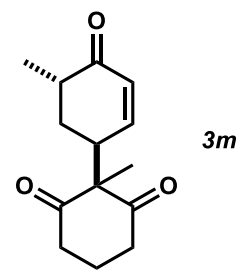

1,5'-Dimethyl-[1,1'-bi(cyclohexan)]-2'-ene-2,4',6-trione (3m): The title compound was prepared according to the General Procedure, using tert-butyldimethyl((6-methylcyclohexa-1,3dien-1-yl)oxy)silane (1c, $56.0 \mathrm{mg}, 0.25 \mathrm{mmol}, 1.0$ equiv) and 2-methylcyclohexane-1,3-dione (2c, $34.6 \mathrm{mg}, 0.275 \mathrm{mmol}, 1.1$ equiv) at $80{ }^{\circ} \mathrm{C}$ for $2 \mathrm{~h}$. Crude ${ }^{1} \mathrm{H}$ NMR prior to purification indicated a 6.6:1 mixture of diastereomers. After purification by flash chromatography $\left(\mathrm{SiO}_{2}\right.$, hexanes/EtOAc $=2: 1$ ), the title compound was isolated as a colorless oil, comprising a 6.6:1 mixture of diastereomers (based on ${ }^{1} \mathrm{H}$ NMR of purified material).

$1^{\text {st }}$ run: $42.7 \mathrm{mg}(73 \%$ yield $)$

$2^{\text {nd }}$ run: $43.8 \mathrm{mg}(75 \%$ yield $)$

TLC $\left(\mathrm{SiO}_{2}\right) R_{f}=0.30$ in 1:1 hexanes/EtOAc, $p$-anisaldehyde stain

${ }^{1} \mathbf{H}$ NMR $\left(400 \mathrm{MHz}, \mathrm{CDCl}_{3}\right) \delta 6.68(\mathrm{~d}, J=10.4 \mathrm{~Hz}, 1 \mathrm{H}), 5.96(\mathrm{dd}, J=10.4,2.6 \mathrm{~Hz}, 1 \mathrm{H}), 3.26$ $(\mathrm{m}, 1 \mathrm{H}), 2.71(\mathrm{~m}, 4 \mathrm{H}), 2.52(\mathrm{~m}, 1 \mathrm{H}), 2.06(\mathrm{~m}, 1 \mathrm{H}), 1.92(\mathrm{~m}, 2 \mathrm{H}), 1.59(\mathrm{~d}, J=13.2 \mathrm{~Hz}, 1 \mathrm{H})$, $1.26(\mathrm{~s}, 3 \mathrm{H}), 1.16(\mathrm{~d}, J=7.4 \mathrm{~Hz}, 3 \mathrm{H})$

${ }^{13}$ C NMR (101 MHz, $\left.\mathrm{CDCl}_{3}\right) \delta$ 209.0, 208.5, 201.5, 147.6, 128.8, 67.8, 39.6, 38.4, 38.0, 37.5, $31.3,17.6,16.3,15.7$

IR (neat) 2965, 2934, 2873, 1723, 1692, 1675, 1458, $1219 \mathrm{~cm}^{-1}$

HRMS $\left(\mathrm{EI}^{+}\right) \mathrm{m} / z$ calc'd for $\mathrm{C}_{14} \mathrm{H}_{18} \mathrm{O}_{3}[\mathrm{M}]^{+}: 234.1267$, found 234.1256<smiles>C[C@H]1CC(=O)C=CC1C1(C)C(=O)CCCC1=O</smiles>

1,6'-Dimethyl-[1,1'-bi(cyclohexan)]-2'-ene-2,4',6-trione (3n): The title compound was prepared according to the General Procedure, using tert-butyldimethyl((5-methylcyclohexa-1,3dien-1-yl)oxy)silane (1e, $56.0 \mathrm{mg}, 0.25 \mathrm{mmol}, 1.0$ equiv) and 2-methylcyclohexane-1,3-dione (2c, $34.6 \mathrm{mg}, 0.275 \mathrm{mmol}, 1.1$ equiv) at $80{ }^{\circ} \mathrm{C}$ for $2 \mathrm{~h}$. Crude ${ }^{1} \mathrm{H}$ NMR prior to purification indicated a $>20: 1$ mixture of diastereomers. After purification by flash chromatography $\left(\mathrm{SiO}_{2}\right.$, hexanes/EtOAc $=2: 1$, the title compound was isolated as a colorless oil, comprising a $>20: 1$ mixture of diastereomers (based on ${ }^{1} \mathrm{H}$ NMR of purified material). 
$1^{\text {st }}$ run: $31.0 \mathrm{mg}(53 \%$ yield $)$

$2^{\text {nd }}$ run: $32.2 \mathrm{mg}(55 \%$ yield $)$

TLC $\left(\mathrm{SiO}_{2}\right) R_{f}=0.40$ in 1:1 hexanes/EtOAc, $p$-anisaldehyde stain

${ }^{1} \mathbf{H}$ NMR $\left(400 \mathrm{MHz}, \mathrm{CDCl}_{3}\right) \delta 6.62(\mathrm{dd}, J=10.4,3.6 \mathrm{~Hz}, 1 \mathrm{H}), 6.09(\mathrm{dd}, J=10.4,2.3 \mathrm{~Hz}, 1 \mathrm{H})$, $3.05(\mathrm{~m}, 1 \mathrm{H}), 2.75(\mathrm{~m}, 4 \mathrm{H}), 2.48(\mathrm{~m}, 1 \mathrm{H}), 2.14(\mathrm{~m}, 3 \mathrm{H}), 1.85(\mathrm{~m}, 1 \mathrm{H}), 1.28(\mathrm{~s}, 3 \mathrm{H}), 1.00(\mathrm{~d}$, $J=6.6 \mathrm{~Hz}, 3 \mathrm{H})$

${ }^{13}$ C NMR $\left(101 \mathrm{MHz}, \mathrm{CDCl}_{3}\right) \delta 209.5,198.1,195.4,146.7,130.5,69.1,47.7,44.0,38.4,37.8$, $31.1,22.1,18.0,16.9$

IR (neat) 2959, 2876, 1724, 1692, $1398 \mathrm{~cm}^{-1}$

HRMS $\left(\mathrm{EI}^{+}\right) \mathrm{m} / z$ calc'd for $\mathrm{C}_{14} \mathrm{H}_{18} \mathrm{O}_{3}[\mathrm{M}]^{+}:$: 234.1251, found 234.1256

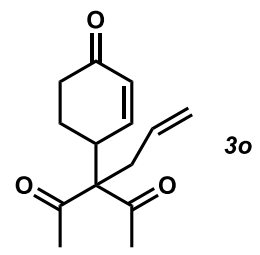

3-Allyl-3-(4-oxocyclohex-2-en-1-yl)pentane-2,4-dione (3o): The title compound was prepared according to the General Procedure, using tert-butyl(cyclohexa-1,3-dien-1-yloxy)dimethylsilane (1b, $52.5 \mathrm{mg}, 0.25 \mathrm{mmol}, 1.0$ equiv) and 3-allylpentane-2,4-dione (2d, $38.5 \mathrm{mg}, 0.275 \mathrm{mmol}, 1.1$ equiv) at $80^{\circ} \mathrm{C}$ for $2 \mathrm{~h}$. After purification by flash chromatography $\left(\mathrm{SiO}_{2}\right.$, hexanes/EtOAc $\left.=1: 1\right)$, the title compound was isolated as a colorless liquid.

$1^{\text {st }}$ run: $37.4 \mathrm{mg}(64 \%$ yield $)$

$2^{\text {nd }}$ run: $38.6 \mathrm{mg}(66 \%$ yield $)$

TLC $\left(\mathrm{SiO}_{2}\right) R_{f}=0.30$ in 1:2 hexanes/EtOAc, $p$-anisaldehyde stain

${ }^{1} \mathbf{H}$ NMR $\left(500 \mathrm{MHz}, \mathrm{CDCl}_{3}\right) \delta 6.89(\mathrm{~d}, J=10.4 \mathrm{~Hz}, 1 \mathrm{H}), 5.98(\mathrm{dd}, J=10.4,2.6 \mathrm{~Hz}, 1 \mathrm{H}), 5.56$ (ddd, $J=17.19 .7,7.3 \mathrm{~Hz}, 1 \mathrm{H}), 5.16(\mathrm{~d}, J=17.1 \mathrm{~Hz}, 1 \mathrm{H}), 5.13(\mathrm{~d}, J=9.7 \mathrm{~Hz}, 1 \mathrm{H}), 3.35$ (d, $J=11.7 \mathrm{~Hz}, 1 \mathrm{H}), 2.83(\mathrm{dd}, J=15.3,7.3 \mathrm{~Hz}, 1 \mathrm{H}), 2.71(\mathrm{dd}, J=15.3,7.3 \mathrm{~Hz}, 1 \mathrm{H}), 2.52(\mathrm{~m}$, $1 \mathrm{H}), 2.38(\mathrm{~m}, 1 \mathrm{H}), 2.17(\mathrm{~s}, 3 \mathrm{H}), 2.14(\mathrm{~s}, 3 \mathrm{H}), 2.00(\mathrm{~m}, 1 \mathrm{H}), 1.67(\mathrm{~m}, 1 \mathrm{H})$

${ }^{13}$ C NMR (126 MHz, $\left.\mathrm{CDCl}_{3}\right) \delta$ 205.5, 204.8, 198.4, 151.6, 131.6, 129.7, 120.1, 72.4, 38.5, 37.8, $34.8,29.1,27.7,25.0$

IR (neat) $2955,1681,1418,1358,1177 \mathrm{~cm}^{-1}$

HRMS $\left(\mathrm{EI}^{+}\right) \mathrm{m} / z$ calc'd for $\mathrm{C}_{14} \mathrm{H}_{19} \mathrm{O}_{3}[\mathrm{M}]^{+}:$: 235.1329, found 235.1334<smiles>CC(=O)C1(C2C=CC(=O)CC2)CCOC1=O</smiles>

3-Acetyl-3-(4-oxocyclohex-2-en-1-yl)dihydrofuran-2(3H)-one (3p): The title compound was prepared according to the General Procedure, using tert-butyl(cyclohexa-1,3-dien-1yloxy)dimethylsilane (1b, $52.5 \mathrm{mg}, 0.25 \mathrm{mmol}, 1.0$ equiv) and 3-acetyl- $\gamma$-butyrolactone (2e, 35.2 mg, $0.275 \mathrm{mmol}, 1.1$ equiv) at $80^{\circ} \mathrm{C}$ for $2 \mathrm{~h}$. Crude ${ }^{1} \mathrm{H}$ NMR prior to purification indicated a 2:1 mixture of diastereomers. After purification by flash chromatography $\left(\mathrm{SiO}_{2}\right.$, hexanes $/ \mathrm{EtOAc}=$ 
1:1), the title compound was isolated as a white solid, comprising a 2:1 mixture of diastereomers (based on ${ }^{1} \mathrm{H}$ NMR of purified material).

$1^{\text {st }}$ run: $47.2 \mathrm{mg}(85 \%$ yield $)$

$2^{\text {nd }}$ run: $48.3 \mathrm{mg}(87 \%$ yield $)$

TLC $\left(\mathrm{SiO}_{2}\right) R_{f}=0.35$ in 1:2 hexanes/EtOAc, $p$-anisaldehyde stain mp $98-99{ }^{\circ} \mathrm{C}$

${ }^{1}$ H NMR $\left(500 \mathrm{MHz}, \mathrm{CDCl}_{3}\right)$ for the major diastereomer: $\delta 6.58(\mathrm{~d}, J=10.3 \mathrm{~Hz}, 1 \mathrm{H}), 6.16(\mathrm{dd}, J$ $=10.3,2.2 \mathrm{~Hz}, 1 \mathrm{H}), 4.35(\mathrm{t}, J=9.1 \mathrm{~Hz}, 1 \mathrm{H}), 4.13(\mathrm{~m}, 1 \mathrm{H}), 3.55(\mathrm{~d}, J=11.2 \mathrm{~Hz}, 1 \mathrm{H}), 2.90$ $(\mathrm{dd}, J=12.8,6.7 \mathrm{~Hz}, 1 \mathrm{H}), 2.51(\mathrm{~m}, 1 \mathrm{H}), 2.44(\mathrm{~m}, 1 \mathrm{H}), 2.41(\mathrm{~s}, 3 \mathrm{H}), 1.97(\mathrm{~m}, 1 \mathrm{H}), 1.85(\mathrm{~m}$, $1 \mathrm{H}), 1.58(\mathrm{~m}, 1 \mathrm{H})$

${ }^{13}$ C NMR (126 MHz, $\left.\mathrm{CDCl}_{3}\right)$ for the major diastereomer: $\delta$ 200.0, 197.4, 174.2, 147.8, 132.3, $66.8,65.5,40.0,36.6,25.5,25.3,25.2$

IR (neat) 2926, 1758, 1710, 1682, 1376, 1255, $1168 \mathrm{~cm}^{-1}$

HRMS $\left(\mathrm{EI}^{+}\right) \mathrm{m} / z$ calc'd for $\mathrm{C}_{12} \mathrm{H}_{15} \mathrm{O}_{4}[\mathrm{M}]^{+}:$: 223.0967, found 223.0970<smiles>O=C1C=CC(C2(C(=O)O)CCCCC2=O)CC1</smiles>

Methyl 2-oxo-1-(4-oxocyclohex-2-en-1-yl)cyclopentane-1-carboxylate (3q): The title compound was prepared according to the General Procedure, using tert-butyl(cyclohexa-1,3dien-1-yloxy)dimethylsilane $(\mathbf{1 b}, \quad 52.5 \mathrm{mg}, \quad 0.25 \mathrm{mmol}, \quad 1.0$ equiv) and methyl 2oxocyclopentane-1-carboxylate (2f, $39.1 \mathrm{mg}, 0.275 \mathrm{mmol}, 1.1$ equiv) at $80{ }^{\circ} \mathrm{C}$ for $2 \mathrm{~h}$. Crude ${ }^{1} \mathrm{H}$ NMR prior to purification indicated a 1.7:1 mixture of diastereomers. After purification by flash chromatography $\left(\mathrm{SiO}_{2}\right.$, hexanes/EtOAc $\left.=2: 1\right)$, the title compound was isolated as a colorless oil, comprising a 1.7:1 mixture of diastereomers (based on ${ }^{1} \mathrm{H}$ NMR of purified material).

$1^{\text {st }}$ run: $45.4 \mathrm{mg}(77 \%$ yield $)$

$2^{\text {nd }}$ run: $46.6 \mathrm{mg}(79 \%$ yield $)$

TLC $\left(\mathrm{SiO}_{2}\right) R_{f}=0.35$ in 1:1 hexanes/EtOAc, $p$-anisaldehyde stain

${ }^{1} \mathbf{H}$ NMR $\left(500 \mathrm{MHz}, \mathrm{CDCl}_{3}\right)$ for the major diastereomer: $\delta 6.45(\mathrm{~d}, J=10.3 \mathrm{~Hz}, 1 \mathrm{H}), 6.02(\mathrm{dd}, J$ $=10.3,2.5 \mathrm{~Hz}, 1 \mathrm{H}), 3.74(\mathrm{~s}, 3 \mathrm{H}), 3.41(\mathrm{~m}, 1 \mathrm{H}), 2.50(\mathrm{~m}, 3 \mathrm{H}), 2.38(\mathrm{~m}, 1 \mathrm{H}), 2.20(\mathrm{~m}, 1 \mathrm{H})$, $2.02(\mathrm{~m}, 3 \mathrm{H}), 1.75(\mathrm{~m}, 2 \mathrm{H})$

${ }^{13} \mathbf{C}$ NMR (126 MHz, $\left.\mathrm{CDCl}_{3}\right)$ for both diastereomers: $\delta 213.5,198.5,170.5,169.3,150.7,149.7$, $131.2,130.2,64.3,62.6,53.1,40.9,40.4,38.7,38.6,37.4,37.2,29.7,29.6,25.7,24.8,19.7$, 19.6

IR (neat) 2954, 1748, 1721, 1680, 1434, 1229, $1110 \mathrm{~cm}^{-1}$

HRMS $\left(\mathrm{EI}^{+}\right) \mathrm{m} / \mathrm{z}$ calc'd for $\mathrm{C}_{13} \mathrm{H}_{16} \mathrm{O}_{4}[\mathrm{M}]^{+}: 236.1044$, found 236.1049 


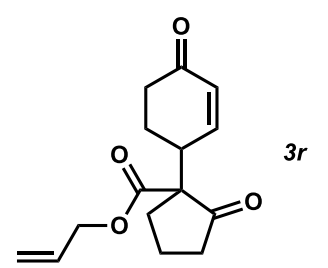

Allyl 2-oxo-1-(4-oxocyclohex-2-en-1-yl)cyclopentane-1-carboxylate (3r): The title compound was prepared according to the General Procedure, using tert-butyl(cyclohexa-1,3-dien-1yloxy)dimethylsilane (1b, $52.5 \mathrm{mg}, 0.25 \mathrm{mmol}, 1.0$ equiv) and allyl 2-oxocyclopentane-1carboxylate (2g, $46.2 \mathrm{mg}, 0.275 \mathrm{mmol}, 1.1$ equiv) at $80{ }^{\circ} \mathrm{C}$ for $2 \mathrm{~h}$. Crude ${ }^{1} \mathrm{H} \mathrm{NMR}$ prior to purification indicated a 1.8:1 mixture of diastereomers. After purification by flash chromatography $\left(\mathrm{SiO}_{2}\right.$, hexanes/EtOAc $\left.=4: 1\right)$, the title compound was isolated as a colorless oil, comprising a 1.8:1 mixture of diastereomers (based on ${ }^{1} \mathrm{H}$ NMR of purified material).

$1^{\text {st }}$ run: $45.2 \mathrm{mg}(69 \%$ yield $)$

$2^{\text {nd }}$ run: $46.5 \mathrm{mg}$ (71\% yield)

TLC $\left(\mathrm{SiO}_{2}\right) R_{f}=0.35$ in 2:1 hexanes/EtOAc, $p$-anisaldehyde stain

${ }^{1} \mathbf{H}$ NMR $\left(500 \mathrm{MHz}, \mathrm{CDCl}_{3}\right)$ for the major diastereomer: $\delta 6.48(\mathrm{~d}, J=10.3 \mathrm{~Hz}, 1 \mathrm{H}), 6.04(\mathrm{dd}, J$ $=10.3,1.6 \mathrm{~Hz}, 1 \mathrm{H}), 5.88(\mathrm{~m}, 1 \mathrm{H}), 5.29(\mathrm{~m}, 2 \mathrm{H}), 4.63(\mathrm{~d}, J=5.6 \mathrm{~Hz}, 2 \mathrm{H}), 3.44(\mathrm{~m}, 1 \mathrm{H})$, 2.64-1.60 (comp. m, 10H)

${ }^{13}$ C NMR (126 MHz, $\mathrm{CDCl}_{3}$ ) for both diastereomers: $\delta$ 213.4, 198.6, 198.5, 169.7, 168.6, 150.8, $149.8,131.1,119.3,66.5,64.3,62.6,40.9,40.4,38.7,38.6,37.4,37.3,29.7,25.7,24.8$, 19.6

IR (neat) 2957, 2887, 1748, 1723, 1681, 1450, 1223, $1148 \mathrm{~cm}^{-1}$

HRMS $\left(\mathrm{EI}^{+}\right) \mathrm{m} / z$ calc'd for $\mathrm{C}_{15} \mathrm{H}_{19} \mathrm{O}_{4}[\mathrm{M}]^{+}: 263.1273$, found 263.1283

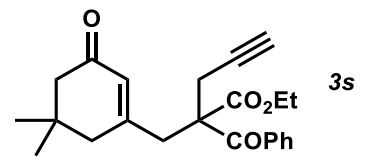

Ethyl 2-benzoyl-2-((5,5-dimethyl-3-oxocyclohex-1-en-1-yl)methyl)pent-4-ynoate (3s): The title compound was prepared according to the General Procedure, using tert-butyl(cyclohexa-1,3dien-1-yloxy)dimethylsilane (1b, $52.5 \mathrm{mg}, 0.25 \mathrm{mmol}, 1.0$ equiv) and ethyl 2-benzoylpent-4ynoate (2h, $63.3 \mathrm{mg}, 0.275 \mathrm{mmol}, 1.1$ equiv) at $80{ }^{\circ} \mathrm{C}$ for $2 \mathrm{~h}$. After purification by flash chromatography $\left(\mathrm{SiO}_{2}\right.$, hexanes/EtOAc $\left.=2: 1\right)$, the title compound was isolated as a colorless liquid.

$1^{\text {st }}$ run: $54.9 \mathrm{mg}(60 \%$ yield $)$

$2^{\text {nd }}$ run: $56.7 \mathrm{mg}(62 \%$ yield $)$

TLC $\left(\mathrm{SiO}_{2}\right) R_{f}=0.35$ in 1:1 hexanes/EtOAc, $p$-anisaldehyde stain

${ }^{1} \mathbf{H}$ NMR $\left(400 \mathrm{MHz}, \mathrm{CDCl}_{3}\right) \delta 7.83(\mathrm{~d}, J=7.6 \mathrm{~Hz}, 2 \mathrm{H}), 7.56(\mathrm{t}, J=7.2 \mathrm{~Hz}, 1 \mathrm{H}), 7.44(\mathrm{t}, J=7.6$ $\mathrm{Hz}, 2 \mathrm{H}), 5.90$ (s, 1H), 4.17 (comp. m, 2H), 3.22 (d, $J=13.9 \mathrm{~Hz}, 1 \mathrm{H}), 3.12$ (d, J=13.9 Hz, $1 \mathrm{H}), 2.96(\mathrm{~s}, 2 \mathrm{H}), 2.15(\mathrm{~s}, 2 \mathrm{H}), 2.09(\mathrm{~s}, 1 \mathrm{H}), 2.01(\mathrm{~s}, 2 \mathrm{H}), 1.10(\mathrm{t}, J=7.1 \mathrm{~Hz}, 3 \mathrm{H}), 0.95(\mathrm{~s}$, $6 \mathrm{H})$

${ }^{13}$ C NMR (101 MHz, CDCl $) \delta 199.4,194.4,171.3,157.3,135.2,133.3,129.3,128.8,128.4$, $78.4,73.4,62.3,60.0,50.8,44.9,40.6,33.9,28.2,28.0,24.1,13.7$

IR (neat) 3277, 2958, 2126, 1732, 1668, 1446, 1368, $1182 \mathrm{~cm}^{-1}$

HRMS $\left(\mathrm{EI}^{+}\right) \mathrm{m} / z$ calc'd for $\mathrm{C}_{23} \mathrm{H}_{27} \mathrm{O}_{4}[\mathrm{M}]^{+}: 367.1905$, found 367.1909 
<smiles>CC1(C)OC(=O)C(C)(C2C=CC(=O)CC2)C(=O)O1</smiles>

2,2,5-Trimethyl-5-(4-oxocyclohex-2-en-1-yl)-1,3-dioxane-4,6-dione (3t): The title compound was prepared according to the General Procedure, using tert-butyl(cyclohexa-1,3-dien-1yloxy)dimethylsilane (1) $52.5 \mathrm{mg}, 0.25 \mathrm{mmol}, 1.0$ equiv) and 2,2,5-trimethyl-1,3-dioxane-4,6dione (2i, $43.5 \mathrm{mg}, 0.275 \mathrm{mmol}, 1.1$ equiv) at $80{ }^{\circ} \mathrm{C}$ for $4 \mathrm{~h}$. After purification by flash chromatography $\left(\mathrm{SiO}_{2}\right.$, hexanes/EtOAc $\left.=1: 1\right)$, the title compound was isolated as a white solid.

$1^{\text {st }}$ run: $34.7 \mathrm{mg}(55 \%$ yield $)$

$2^{\text {nd }}$ run: $35.9 \mathrm{mg}(57 \%$ yield $)$

TLC $\left(\mathrm{SiO}_{2}\right) R_{f}=0.35$ in 1:2 hexanes/EtOAc, $p$-anisaldehyde stain mp $98-99{ }^{\circ} \mathrm{C}$

${ }^{1}$ H NMR $\left(500 \mathrm{MHz}, \mathrm{CDCl}_{3}\right) \delta 7.09(\mathrm{~d}, J=10.4 \mathrm{~Hz}, 1 \mathrm{H}), 6.08(\mathrm{~d}, J=10.4 \mathrm{~Hz}, 1 \mathrm{H}), 3.17(\mathrm{~d}, J=$ $11.2 \mathrm{~Hz}, 1 \mathrm{H}), 2.53(\mathrm{~d}, J=16.7 \mathrm{~Hz}, 1 \mathrm{H}), 2.36(\mathrm{~m}, 1 \mathrm{H}), 2.05(\mathrm{~m}, 1 \mathrm{H}), 1.85(\mathrm{~m}, 1 \mathrm{H}), 1.76(\mathrm{~s}$, $6 \mathrm{H})$

${ }^{13}$ C NMR (126 MHz, $\left.\mathrm{CDCl}_{3}\right) \delta 197.6,169.5,168.4,147.0,130.3,105.5,52.0,45.8,37.0,30.2$, $28.2,26.0,21.2$

IR (neat) 2950, 1767, 1731, 1676, 1120, 972, $767 \mathrm{~cm}^{-1}$

HRMS $\left(\mathrm{EI}^{+}\right) \mathrm{m} / z$ calc'd for $\mathrm{C}_{13} \mathrm{H}_{17} \mathrm{O}_{5}[\mathrm{M}]^{+}: 253.1074$, found 253.1076

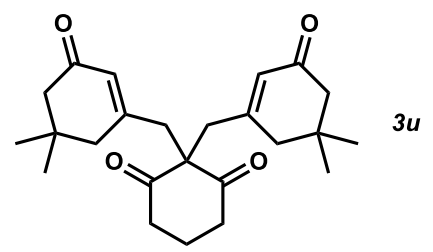

2,2-Bis((5,5-dimethyl-3-oxocyclohex-1-en-1-yl)methyl)cyclohexane-1,3-dione (3u): The title compound was prepared according to the general procedure using crude tert-butyl $((5,5$-dimethyl3-methylenecyclohex-1-en-1-yl)oxy)dimethylsilane (1a, prepared according to ref 3) (63 mg, $0.25 \mathrm{mmol}, 2.0$ equiv) and cyclohexane-1,3-dione (2a, $14.0 \mathrm{mg}, 0.125 \mathrm{mmol}, 1.0$ equiv) at $80{ }^{\circ} \mathrm{C}$ for $2 \mathrm{~h}$. After purification by flash chromatography $\left(\mathrm{SiO}_{2}\right.$, hexanes/EtOAc $\left.=1: 1\right)$, the title compound was isolated as a white solid.

$1^{\text {st }}$ run: $38.9 \mathrm{mg}(81 \%$ yield $)$

$2^{\text {nd }}$ run: $37.9 \mathrm{mg}(79 \%$ yield $)$

TLC $\left(\mathrm{SiO}_{2}\right) R_{f}=0.25$ in 1:2 hexanes/EtOAc, $p$-anisaldehyde stain mp $118-119^{\circ} \mathrm{C}$

${ }^{1} \mathbf{H}$ NMR $\left(400 \mathrm{MHz}, \mathrm{CDCl}_{3}\right) \delta 5.55(\mathrm{~s}, 2 \mathrm{H}), 2.71(\mathrm{t}, J=6.7 \mathrm{~Hz}, 4 \mathrm{H}), 2.67(\mathrm{~s}, 4 \mathrm{H}), 2.15(\mathrm{~s}, 4 \mathrm{H})$, $2.09(\mathrm{~s}, 4 \mathrm{H}), 2.03(\mathrm{~m}, 2 \mathrm{H}), 0.99(\mathrm{~s}, 12 \mathrm{H})$

${ }^{13}$ C NMR (101 MHz, $\left.\mathrm{CDCl}_{3}\right) \delta$ 208.1, 199.1, 157.5, 128.0, 67.5, 50.7, 45.6, 43.6, 38.8, 33.7, $28.2,16.9$

IR (neat) 2957, 1724, 1695, 1664, $1279 \mathrm{~cm}^{-1}$

HRMS $\left(\mathrm{EI}^{+}\right) \mathrm{m} / z$ calc'd for $\mathrm{C}_{24} \mathrm{H}_{32} \mathrm{O}_{4}[\mathrm{M}]^{+}:$384.2296, found 384.2301 


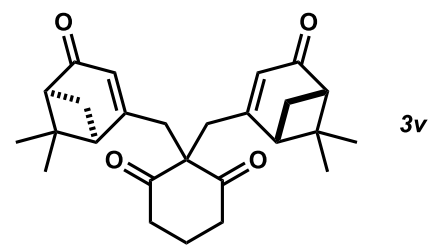

2,2-Bis(((1R,5S)-6,6-dimethyl-4-oxobicyclo[3.1.1]hept-2-en-2-yl)methyl)cyclohexane-1,3dione (3v): The title compound was prepared according to the general procedure using crude tert-butyl(((1S,5R)-6,6-dimethyl-4-methylenebicyclo[3.1.1]hept-2-en-2-yl)oxy)dimethylsilane $\mathbf{1 h}$ (prepared according to ref 3) $(66.0 \mathrm{mg}, 0.25 \mathrm{mmol}, 2.0$ equiv) and cyclohexane-1,3-dione (2a, $14.0 \mathrm{mg}, 0.125 \mathrm{mmol}, 1.0$ equiv) at $80{ }^{\circ} \mathrm{C}$ for $2 \mathrm{~h}$. After purification by flash chromatography $\left(\mathrm{SiO}_{2}\right.$, hexanes/EtOAc $\left.=1: 1\right)$, the title compound was isolated as a white solid.

$1^{\text {st }}$ run: $38.76 \mathrm{mg}(76 \%$ yield $)$

$2^{\text {nd }}$ run: $40.8 \mathrm{mg}$ ( $80 \%$ yield $)$

TLC $\left(\mathrm{SiO}_{2}\right) R_{f}=0.25$ in 1:2 hexanes/EtOAc, $p$-anisaldehyde stain

mp $160-162^{\circ} \mathrm{C}$

${ }^{1} \mathbf{H}$ NMR $\left(400 \mathrm{MHz}, \mathrm{CDCl}_{3}\right) \delta 5.38(\mathrm{~s}, 2 \mathrm{H}), 2.77$ (comp. m, 10H), $2.60(\mathrm{t}, J=5.0 \mathrm{~Hz}, 2 \mathrm{H}), 2.28$ $(\mathrm{t}, J=5.8 \mathrm{~Hz}, 2 \mathrm{H}), 2.08(\mathrm{~m}, 2 \mathrm{H}), 2.02(\mathrm{~d}, J=9.4 \mathrm{~Hz}, 2 \mathrm{H}), 1.46(\mathrm{~s}, 6 \mathrm{H}), 0.95(\mathrm{~s}, 6 \mathrm{H})$

${ }^{13} \mathbf{C}$ NMR $\left(101 \mathrm{MHz}, \mathrm{CDCl}_{3}\right) \delta 207.3,202.9,166.7,123.8,67.4,57.4,54.2,50.1,42.9,38.3$, $26.6,22.1,17.2$

IR (neat) 2954, 1725, 1675, 1283, $1240 \mathrm{~cm}^{-1}$

HRMS $\left(\mathrm{EI}^{+}\right) \mathrm{m} / z$ calc'd for $\mathrm{C}_{26} \mathrm{H}_{32} \mathrm{O}_{4}[\mathrm{M}]^{+}:$408.2309, found 408.2301

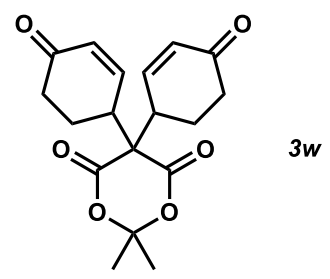

2,2-Dimethyl-5,5-bis(4-oxocyclohex-2-en-1-yl)-1,3-dioxane-4,6-dione (3w): The title compound was prepared according to the general procedure using tert-butyl(cyclohexa-1,3-dien1-yloxy)dimethylsilane (1b, $52.5 \mathrm{mg}, 0.25 \mathrm{mmol}, 2.0$ equiv) and 2,2-dimethyl-1,3-dioxane-4,6dione (2j, $18.0 \mathrm{mg}, 0.125 \mathrm{mmol}, 1.0$ equiv) at $80{ }^{\circ} \mathrm{C}$ for $2 \mathrm{~h}$. Crude ${ }^{1} \mathrm{H}$ NMR prior to purification indicated a 1:1 mixture of diastereomers. After purification by flash chromatography $\left(\mathrm{SiO}_{2}\right.$, hexanes/EtOAc $=2: 1$ ), the title compound was isolated as a white solid as a 1:1 mixture of diastereomers (based on ${ }^{1} \mathrm{H}$ NMR of purified material).

$1^{\text {st }}$ run: $26.1 \mathrm{mg}(63 \%$ yield $)$

$2^{\text {nd }}$ run: $27.0 \mathrm{mg}$ (65\% yield)

TLC $\left(\mathrm{SiO}_{2}\right) R_{f}=0.32$ in 1:1 hexanes/EtOAc, $p$-anisaldehyde stain

mp $215-217^{\circ} \mathrm{C}$

${ }^{1}$ H NMR $\left(400 \mathrm{MHz}, \mathrm{CDCl}_{3}\right) \delta 7.14(\mathrm{~d}, J=10.5 \mathrm{~Hz}, 1 \mathrm{H}), 6.89$ (d, $\left.J=10.5 \mathrm{~Hz}, 1 \mathrm{H}\right), 6.14$ (app. d, $J=10.5 \mathrm{~Hz}, 2 \mathrm{H}), 3.54(\mathrm{~m}, 2 \mathrm{H}), 2.62(\mathrm{~m}, 2 \mathrm{H}), 2.44(\mathrm{~m}, 2 \mathrm{H}), 2.11(\mathrm{~m}, 3 \mathrm{H}), 1.87(\mathrm{~m}, 1 \mathrm{H})$, $1.80(\mathrm{~s}, 3 \mathrm{H}), 1.78(\mathrm{~s}, 3 \mathrm{H})$

${ }^{13}$ C NMR $\left(101 \mathrm{MHz}, \mathrm{CDCl}_{3}\right) \delta$ 196.8, 166.1, 145.5, 145.4, 131.4, 130.9, 107.2, 59.7, 41.1, 40.0, 37.2, 37.1, 30.2, 30.1, 27.4, 25.6

IR (neat) 2953, 2924, 2869, 1731, 1713, 1674, 1392, 1204, 1061, $816 \mathrm{~cm}^{-1}$ 
HRMS $\left(\mathrm{ES}^{+}\right) \mathrm{m} / \mathrm{z}$ calc'd for $\mathrm{C}_{18} \mathrm{H}_{20} \mathrm{O}_{6}[\mathrm{M}+\mathrm{Na}]^{+} 355.1158$, found 355.1151

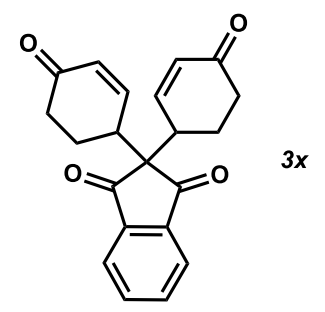

2,2-Bis(4-oxocyclohex-2-en-1-yl)-1H-indene-1,3(2H)-dione (3x): The title compound was prepared according to the general procedure using tert-butyl(cyclohexa-1,3-dien-1yloxy)dimethylsilane (1) $\mathbf{1 b}, 5 \mathrm{mg}, 0.25 \mathrm{mmol}, 2.0$ equiv) and $1 \mathrm{H}$-indene-1,3(2H)-dione (2k, $18.3 \mathrm{mg}, 0.125 \mathrm{mmol}, 1.0$ equiv) at $80^{\circ} \mathrm{C}$ for $2 \mathrm{~h}$. Crude ${ }^{1} \mathrm{H}$ NMR prior to purification indicated a 2.6:1 mixture of diastereomers. After purification by flash chromatography $\left(\mathrm{SiO}_{2}\right.$, hexanes/EtOAc $=2: 1$ ), the title compound was isolated as a colorless oil, comprising a 2.6:1 mixture of diastereomers (based on ${ }^{1} \mathrm{H}$ NMR of purified material).

$1^{\text {st }}$ run: $29.6 \mathrm{mg}(71 \%$ yield $)$

$2^{\text {nd }}$ run: $28.8 \mathrm{mg}(69 \%$ yield $)$

TLC $\left(\mathrm{SiO}_{2}\right) R_{f}=0.30$ in 1:1 hexanes/EtOAc, $p$-anisaldehyde stain

${ }^{1}$ H NMR (500 MHz, $\mathrm{CDCl}_{3}$ ) for the major diastereomer: $\delta 8.00$ (br. s, 2H), 7.93 (br. s, 2H), 6.73 $(\mathrm{d}, J=10.0 \mathrm{~Hz}, 2 \mathrm{H}), 6.01(\mathrm{~d}, J=10.0 \mathrm{~Hz}, 2 \mathrm{H}), 3.38(\mathrm{~s}, 2 \mathrm{H}), 2.51(\mathrm{~m}, 2 \mathrm{H}), 2.35(\mathrm{~m}, 4 \mathrm{H})$, $1.99(\mathrm{~m}, 4 \mathrm{H})$

${ }^{13}$ C NMR (126 MHz, $\mathrm{CDCl}_{3}$ ) for both diastereomers: $\delta$ 202.2, 201.7, 201.6, 197.6, 147.0, 142.4, $142.2,136.9,136.7,131.5,131.3,123.5,123.3,123.2,60.5,38.5,38.3,37.3,25.9,24.9$

IR (neat) 2953, 2925, 2854, 1736, 1702, 1682, 1233, $772 \mathrm{~cm}^{-1}$

HRMS $\left(\mathrm{EI}^{+}\right) m / z$ calc'd for $\mathrm{C}_{21} \mathrm{H}_{18} \mathrm{O}_{4}[\mathrm{M}]^{+}:$334.1209, found 334.1205

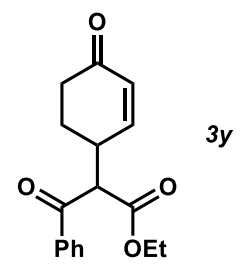

Ethyl 3-oxo-2-(4-oxocyclohex-2-en-1-yl)-3-phenylpropanoate (3y): The title compound was prepared according to the General Procedure, using tert-butyl(cyclohexa-1,3-dien-1yloxy)dimethylsilane (1b, $52.5 \mathrm{mg}, 0.25 \mathrm{mmol}, 1.0$ equiv) and ethyl 3-oxo-3-phenylpropanoate (21, $52.8 \mathrm{mg}, 0.275 \mathrm{mmol}, 1.1$ equiv) at $80{ }^{\circ} \mathrm{C}$ for $4 \mathrm{~h}$. Crude ${ }^{1} \mathrm{H}$ NMR prior to purification indicated a 1.2:1 mixture of diastereomers. After purification by flash chromatography $\left(\mathrm{SiO}_{2}\right.$, hexanes/EtOAc $=4: 1)$, the title compound was isolated as a colorless oil, comprising a 1.2:1 mixture of diastereomers (based on ${ }^{1} \mathrm{H}$ NMR of purified material).

$1^{\text {st }}$ run: $28.6 \mathrm{mg}(40 \%$ yield $)$

$2^{\text {nd }}$ run: $25.7 \mathrm{mg}(36 \%$ yield $)$

TLC $\left(\mathrm{SiO}_{2}\right) R_{f}=0.60$ in 1:1 hexanes/EtOAc, $p$-anisaldehyde stain

${ }^{1} \mathbf{H}$ NMR $\left(400 \mathrm{MHz}, \mathrm{CDCl}_{3}\right.$ ) for both diastereomers: $\delta 8.02$ (app. d, $\left.J=7.5 \mathrm{~Hz}, 2 \mathrm{H}\right), 7.62(\mathrm{~m}$, 1H), $7.50(\mathrm{~m}, 2 \mathrm{H}), 6.99(\mathrm{~d}, J=10.3 \mathrm{~Hz}, 0.5 \mathrm{H}), 6.84(\mathrm{~d}, J=10.3 \mathrm{~Hz}, 0.5 \mathrm{H}), 6.06(\mathrm{dd}, J=$ 
$10.3,2.3 \mathrm{~Hz}, 0.5 \mathrm{H}), 5.99$ (dd, $J=10.3,2.1 \mathrm{~Hz}, 0.5 \mathrm{H}), 4.17$ (app. q, $J=7.4 \mathrm{~Hz}, 2 \mathrm{H}), 3.49$ $(\mathrm{m}, 1 \mathrm{H}), 2.46(\mathrm{~m}, 3 \mathrm{H}), 1.86(\mathrm{~m}, 1 \mathrm{H}), 1.17(\mathrm{~m}, 3 \mathrm{H})$

${ }^{13}$ C NMR (101 MHz, $\left.\mathrm{CDCl}_{3}\right)$ for both diastereomers: $\delta$ 198.6, 193.3, 193.1, 168.0, 151.2, 150.9, $134.1,130.2,130.0,129.4,128.9,128.6,127.6,79.8,62.0,61.9,57.6,27.3,36.8,36.1,36.0$, $27.4,27.0,14.0$

IR (neat) 2979, 2937, 1732, 1680, 1390, $1205 \mathrm{~cm}^{-1}$

HRMS $\left(\mathrm{EI}^{+}\right) \mathrm{m} / z$ calc'd for $\mathrm{C}_{17} \mathrm{H}_{18} \mathrm{O}_{4}[\mathrm{M}]^{+}: 286.1207$, found 286.1205<smiles>CC(=O)C(C(=O)Br)C1C=CC(=O)CC1</smiles>

5,5-Dimethyl-3-(4-oxocyclohex-2-en-1-yl)hexane-2,4-dione (3z): The title compound was prepared according to the General Procedure, using tert-butyl(cyclohexa-1,3-dien-1yloxy)dimethylsilane (1b, $52.5 \mathrm{mg}, 0.25 \mathrm{mmol}, 1.0$ equiv) and 5,5-dimethylhexane-2,4-dione (2m, $39.0 \mathrm{mg}, 0.275 \mathrm{mmol}, 1.1$ equiv) at $80{ }^{\circ} \mathrm{C}$ for $2 \mathrm{~h}$. Crude ${ }^{1} \mathrm{H}$ NMR prior to purification indicated a 1:1 mixture of diastereomers. After purification by flash chromatography $\left(\mathrm{SiO}_{2}\right.$, hexanes/EtOAc $=2: 1)$, the two diastereomers were isolated as colorless oils.

$1^{\text {st }}$ run: $37.2 \mathrm{mg}(63 \%$ combined yield $)$

$2^{\text {nd }}$ run: $36.0 \mathrm{mg}(61 \%$ combined yield $)$

Less polar diastereomer:

TLC $\left(\mathrm{SiO}_{2}\right) R_{f}=0.40$ in 1:1 hexanes/EtOAc, $p$-anisaldehyde stain

${ }^{1} \mathbf{H}$ NMR $\left(500 \mathrm{MHz}, \mathrm{CDCl}_{3}\right) \delta 6.63(\mathrm{~d}, J=9.2 \mathrm{~Hz}, 1 \mathrm{H}), 6.04(\mathrm{~d}, J=9.2 \mathrm{~Hz}, 1 \mathrm{H}), 4.09(\mathrm{~d}, J=$ $10.4 \mathrm{~Hz}, 1 \mathrm{H}), 3.34$ (br. s, 1H), $2.43(\mathrm{~m}, 3 \mathrm{H}), 2.20(\mathrm{~s}, 3 \mathrm{H}), 1.98(\mathrm{~m}, 1 \mathrm{H}), 1.14(\mathrm{~s}, 9 \mathrm{H})$

${ }^{13}$ C NMR (126 MHz, $\left.\mathrm{CDCl}_{3}\right) \delta$ 209.5, 202.9, 198.1, 149.2, 130.8, 66.9, 46.3, 37.6, 36.8, 27.9, $27.6,25.9$

IR (neat) 2957, 2872, 1722, 1689, 1366, $1163 \mathrm{~cm}^{-1}$

HRMS $\left(\mathrm{EI}^{+}\right) \mathrm{m} / z$ calc'd for $\mathrm{C}_{14} \mathrm{H}_{21} \mathrm{O}_{3}[\mathrm{M}]^{+}: 237.1495$, found 237.1491

More polar diastereomer:

TLC $\left(\mathrm{SiO}_{2}\right) R_{f}=0.35$ in 1:1 hexanes/EtOAc, $p$-anisaldehyde stain

${ }^{1} \mathbf{H}$ NMR $\left(500 \mathrm{MHz}, \mathrm{CDCl}_{3}\right) \delta 6.56(\mathrm{dd}, J=10.2,2.2 \mathrm{~Hz}, 1 \mathrm{H}), 5.99(\mathrm{dd}, J=10.2,2.0 \mathrm{~Hz}, 1 \mathrm{H})$, $4.10(\mathrm{~d}, J=10.3 \mathrm{~Hz}, 1 \mathrm{H}), 3.35(\mathrm{~m}, 1 \mathrm{H}), 2.53(\mathrm{~m}, 1 \mathrm{H}), 2.40(\mathrm{~m}, 1 \mathrm{H}), 2.19(\mathrm{~s}, 3 \mathrm{H}), 1.98(\mathrm{~m}$, $1 \mathrm{H}), 1.73(\mathrm{~m}, 1 \mathrm{H}), 1.14(\mathrm{~s}, 9 \mathrm{H})$

${ }^{13}$ C NMR (101 MHz, $\left.\mathrm{CDCl}_{3}\right) \delta$ 209.8, 202.3, 198.0, 150.0, 130.2, 66.4, 46.1, 36.9, 36.0, 27.2, $26.5,25.9$

IR (neat) 2965, 2931, 2872, 1721, 1690, 1680, 1366, $994 \mathrm{~cm}^{-1}$

HRMS $\left(\mathrm{EI}^{+}\right) \mathrm{m} / z$ calc'd for $\mathrm{C}_{14} \mathrm{H}_{20} \mathrm{O}_{3}[\mathrm{M}]^{+}: 236.1415$, found 236.1413 


\section{Procedures and Data for Synthetic Applications}
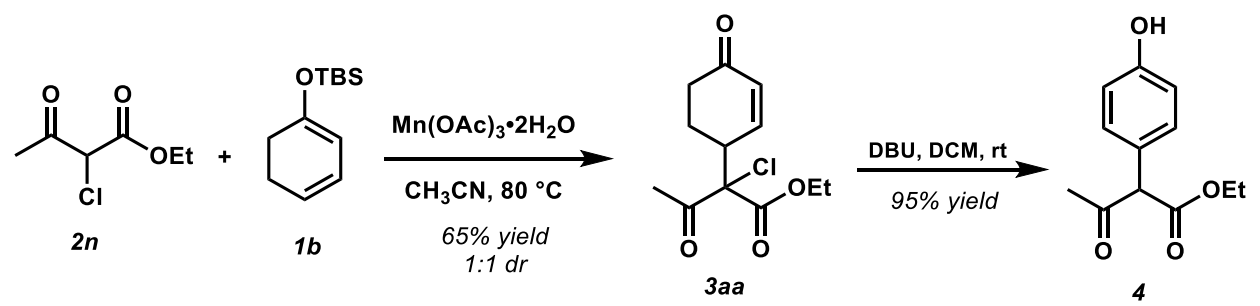

Ethyl 2-chloro-3-oxo-2-(4-oxocyclohex-2-en-1-yl)butanoate (3aa): The title compound was prepared according to the General Procedure, using tert-butyl(cyclohexa-1,3-dien-1yloxy)dimethylsilane (1b, $52.5 \mathrm{mg}, 0.25 \mathrm{mmol}, 1.0$ equiv) and ethyl 2-chloro-3-oxobutanoate (2n, $45.1 \mathrm{mg}, 0.275 \mathrm{mmol}, 1.1$ equiv) at $80{ }^{\circ} \mathrm{C}$ for $2 \mathrm{~h}$. Crude ${ }^{1} \mathrm{H}$ NMR prior to purification indicated a 1:1 mixture of diastereomers. After purification by flash chromatography $\left(\mathrm{SiO}_{2}\right.$, hexanes/EtOAc $=2: 1$, the title compound was isolated as a colorless oil, comprising a 1:1 mixture of diastereomers (based on ${ }^{1} \mathrm{H}$ NMR of purified material).

$1^{\text {st }}$ run: $41.3 \mathrm{mg}(64 \%$ yield $)$

$2^{\text {nd }}$ run: $42.6 \mathrm{mg}(66 \%$ yield $)$

TLC $\left(\mathrm{SiO}_{2}\right) R_{f}=0.35$ in 1:1 hexanes/EtOAc, $p$-anisaldehyde stain

${ }^{1}$ H NMR $\left(500 \mathrm{MHz}, \mathrm{CDCl}_{3}\right) \delta 6.81(\mathrm{~d}, J=10.4 \mathrm{~Hz}, 1 \mathrm{H}), 6.73(\mathrm{~d}, J=10.4 \mathrm{~Hz}, 1 \mathrm{H}), 6.04(\mathrm{~d}, J=$ $10.4 \mathrm{~Hz}, 2 \mathrm{H}), 4.31(\mathrm{~m}, 4 \mathrm{H}), 3.62(\mathrm{~m}, 1 \mathrm{H}), 3.57(\mathrm{~m}, 1 \mathrm{H}), 2.54(\mathrm{~m}, 2 \mathrm{H}), 2.42(\mathrm{~m}, 2 \mathrm{H}), 2.39$ $(\mathrm{s}, 3 \mathrm{H}), 2.36(\mathrm{~s}, 3 \mathrm{H}), 2.11(\mathrm{~m}, 1 \mathrm{H}), 1.96(\mathrm{~m}, 3 \mathrm{H}), 1.30(\mathrm{~m}, 6 \mathrm{H})$

${ }^{13}$ C NMR (126 MHz, $\left.\mathrm{CDCl}_{3}\right) \delta 198.1,198.0,197.9,196.8,166.1,165.7,148.6,148.4,130.5$, $63.8,63.6,42.7,41.7,36.9,36.8,26.9,26.1,24.7,13.9$

IR (neat) 2982, 1724, 1682, 1417, 1174, $1044 \mathrm{~cm}^{-1}$

HRMS $\left(\mathrm{EI}^{+}\right) \mathrm{m} / z$ calc'd for $\mathrm{C}_{12} \mathrm{H}_{15} \mathrm{O}_{4} \mathrm{Cl}[\mathrm{M}]^{+}: 258.0660$, found 258.0659

\section{Ethyl 2-(4-hydroxyphenyl)-3-oxobutanoate (4)}

To a solution of ethyl 2-chloro-3-oxo-2-(4-oxocyclohex-2-en-1-yl)butanoate 3aa (51.6 mg, 0.2 mmol, 1.0 equiv) in dichloromethane $(4 \mathrm{~mL})$ was added DBU (45.6 mg, $0.3 \mathrm{mmol}, 1.5 \mathrm{equiv}$ ). The mixture was stirred for $1 \mathrm{~h}$ at room temperature at which time TLC showed total consumption of the starting material. Deionized water $(5.0 \mathrm{~mL})$ was added and the mixture was poured into a separatory funnel. The aq phase was extracted with dichloromethane $(3 \times 5.0 \mathrm{~mL})$. The organic layers were combined, dried over $\mathrm{Na}_{2} \mathrm{SO}_{4}$, filtered, and concentrated under reduced pressure. After purification by flash chromatography $\left(\mathrm{SiO}_{2}\right.$, hexanes/EtOAc $\left.=2: 1\right)$, the title compound was isolated as a colorless liquid 4 (44.4 $\mathrm{mg}, 95 \%$ yield).

TLC $\left(\mathrm{SiO}_{2}\right) R_{f}=0.45$ in 1:1 hexanes/EtOAc, $p$-anisaldehyde stain

${ }^{1} \mathbf{H}$ NMR $\left(500 \mathrm{MHz}, \mathrm{CDCl}_{3}\right) \delta 7.19(\mathrm{~d}, J=8.5 \mathrm{~Hz}, 2 \mathrm{H}), 6.81(\mathrm{~d}, J=8.5 \mathrm{~Hz}, 2 \mathrm{H}), 5.44$ (br. s, 1H), $4.63(\mathrm{~s}, 1 \mathrm{H}), 4.20(\mathrm{~m}, 2 \mathrm{H}), 2.18(\mathrm{~s}, 3 \mathrm{H}), 1.28(\mathrm{~m}, 3 \mathrm{H})$

${ }^{13}$ C NMR $\left(126 \mathrm{MHz}, \mathrm{CDCl}_{3}\right) \delta$ 202.3, 169.0, 155.8, 132.4, 130.6, 124.6, 115.9, 115.0, 64.9, 61.7, $28.7,14.1$

IR (neat) 3416, 2982, 1712, 1638, 1612, 1514, 1147, $838 \mathrm{~cm}^{-1}$

HRMS $\left(\mathrm{EI}^{+}\right) \mathrm{m} / z$ calc'd for $\mathrm{C}_{12} \mathrm{H}_{14} \mathrm{O}_{4}[\mathrm{M}]^{+}: 222.0900$, found 222.0892 


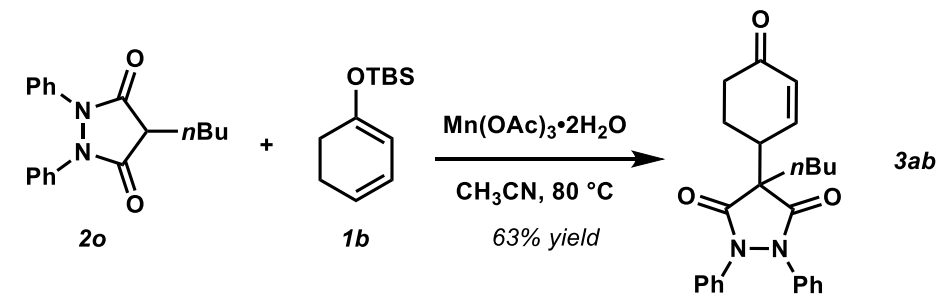

4-Butyl-4-(4-oxocyclohex-2-en-1-yl)-1,2-diphenylpyrazolidine-3,5-dione: The title compound was prepared according to the General Procedure, using tert-butyl(cyclohexa-1,3-dien-1yloxy)dimethylsilane (1b, $52.5 \mathrm{mg}, 0.25 \mathrm{mmol}, 1.0$ equiv) and phenylbutazone (2o, $84.7 \mathrm{mg}$, $0.275 \mathrm{mmol}, 1.1$ equiv) at $80{ }^{\circ} \mathrm{C}$ for $2 \mathrm{~h}$. After purification by flash chromatography $\left(\mathrm{SiO}_{2}\right.$, hexanes/EtOAc $=1: 1)$, the title compound was isolated as a colorless liquid.

$1^{\text {st }}$ run: $64.3 \mathrm{mg}(64 \%$ yield $)$

$2^{\text {nd }}$ run: $62.3 \mathrm{mg}(62 \%$ yield $)$

TLC $\left(\mathrm{SiO}_{2}\right) R_{f}=0.25$ in 1:2 hexanes/EtOAc, $p$-anisaldehyde stain

${ }^{1} \mathbf{H}$ NMR $\left(400 \mathrm{MHz}, \mathrm{CDCl}_{3}\right) \delta 7.32(\mathrm{~m}, 8 \mathrm{H}), 7.20(\mathrm{~m}, 2 \mathrm{H}), 7.10(\mathrm{~d}, J=10.4 \mathrm{~Hz}, 1 \mathrm{H}), 6.10(\mathrm{dd}, J$ $=10.4,2.3 \mathrm{~Hz}, 1 \mathrm{H}), 3.05(\mathrm{~m}, 1 \mathrm{H}), 2.54(\mathrm{~d}, J=16.9 \mathrm{~Hz}, 1 \mathrm{H}), 2.37(\mathrm{~m}, 1 \mathrm{H}), 2.15(\mathrm{~m}, 2 \mathrm{H})$, $2.01(\mathrm{~m}, 3 \mathrm{H}), 1.35(\mathrm{~m}, 4 \mathrm{H}), 0.88(\mathrm{t}, J=6.7 \mathrm{~Hz}, 3 \mathrm{H})$

${ }^{13}$ C NMR (101 MHz, CDCl 3$) \delta 198.1,171.6,147.4,135.1,131.1,129.1,127.1,122.5,56.0,42.3$, $37.1,33.6,26.8,25.1,22.7,15.7$

IR (neat) 2957, 2927, 2871, 1751, 1714, 1682, 1493, 1292, $731 \mathrm{~cm}^{-1}$

HRMS $\left(\mathrm{EI}^{+}\right) \mathrm{m} / z$ calc'd for $\mathrm{C}_{25} \mathrm{H}_{26} \mathrm{O}_{3} \mathrm{~N}_{2}[\mathrm{M}]^{+}:$: 402.1946, found 402.1944

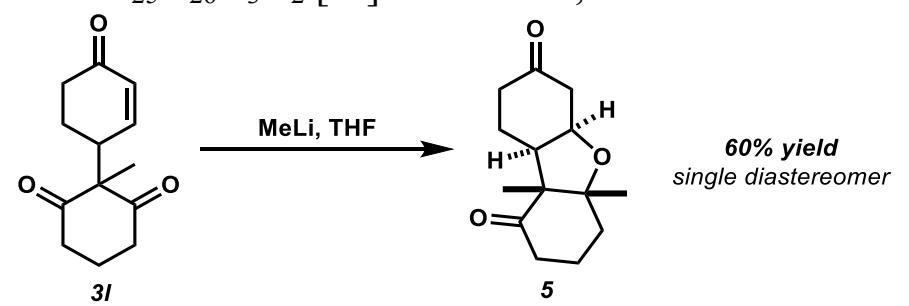

\section{4a,9b-dimethyldecahydrodibenzo[b,d]furan-1,7-dione (5)}

The title compound was prepared according to follow procedure: 1-methyl-[1,1'-bi(cyclohexan)]2'-ene-2,4',6-trione (31, $44.0 \mathrm{mg}, 0.2 \mathrm{mmol}, 1.0$ equiv) was weighed into a flame-dried $25 \mathrm{~mL}$ flask equipped with a magnetic stir bar. The flask was sealed with a rubber septum and then it was evacuated and backfilled with nitrogen (three cycles). The flask was charged with dry THF $(4.0 \mathrm{~mL})$ and the resulting mixture was cooled to $-78^{\circ} \mathrm{C}$. A solution of $\mathrm{MeLi}$ in $\mathrm{Et}_{2} \mathrm{O}(80 \mu \mathrm{L}$, 3.0 M solution, $0.24 \mathrm{mmol}, 1.2$ equiv) was added dropwise over $2 \mathrm{~min}$ and then the mixture was stirred at $-78{ }^{\circ} \mathrm{C}$ for $30 \mathrm{~min}$. The mixture was slowly warmed to room temperature and stirred for another $30 \mathrm{~min}$. The reaction was quenched with water $(3 \mathrm{~mL})$ and then extracted with EtOAc $(3 \times 10 \mathrm{~mL})$. The combined organic layers were dried over anhydrous $\mathrm{Na}_{2} \mathrm{SO}_{4}$, filtered, and concentrated in vacuo to obtain a yellow oil. After purification by flash chromatography $\left(\mathrm{SiO}_{2}\right.$, hexanes/EtOAc $\left.=4: 1\right)$, Compound 5 was isolated as colorless oil $(28.3 \mathrm{mg}, 60 \%$ yield $)$.

TLC $\left(\mathrm{SiO}_{2}\right) R_{f}=0.25$ in 1:1 hexanes/EtOAc, $p$-anisaldehyde stain

${ }^{1}$ H NMR $\left(500 \mathrm{MHz}, \mathrm{CDCl}_{3}\right) \delta 4.28(\mathrm{~m}, 1 \mathrm{H}), 3.02(\mathrm{~m}, 1 \mathrm{H}), 2.73(\mathrm{~m}, 1 \mathrm{H}), 2.48(\mathrm{~m}, 5 \mathrm{H}), 2.11(\mathrm{~m}$, $1 \mathrm{H}), 1.94(\mathrm{~m}, 3 \mathrm{H}), 1.78(\mathrm{~m}, 2 \mathrm{H}), 1.31(\mathrm{~s}, 3 \mathrm{H}), 1.13(\mathrm{~s}, 3 \mathrm{H})$

${ }^{13}$ C NMR $\left(126 \mathrm{MHz}_{\mathrm{CDCl}}\right) \delta 213.3,210.2,87.0,73.1,60.3,44.7,44.4,38.5,37.0,34.7,24.1$, $21.3,19.6,15.7$ 
IR (neat) 2941, 2876, 1714, 1698, 1458, 1103, $1040 \mathrm{~cm}^{-1}$

HRMS $\left(\mathrm{EI}^{+}\right) \mathrm{m} / z$ calc'd for $\mathrm{C}_{14} \mathrm{H}_{20} \mathrm{O}_{3}[\mathrm{M}]^{+}: 236.1415$, found 236.1413

\section{References}

1. Pangborn, A. B.; Giardello, M. A.; Grubbs, R. H.; Rosen, R. K.; Timmers, F. J. Organometallics 1996, 15, 1518-1520.

2. Heiba, E. I.; Dessau, R. M.; Koehl, W. J., Jr. J. Am. Chem. Soc. 1969, 91, 138-145.

3. $\quad$ Liu, X.; Chen, X.; Mohr, J. T. Org. Lett. 2015, 17, 3572-3575.

4. Still, W. C.; Kahn, M.; Mitra, A. J. Org. Chem. 1978, 43, 2923-2925. 